\title{
Гигантская отрицательная фотопроводимость пленок PbSnTe: In с краем чувствительности вблизи 30 мкм
}

\author{
(С) А.Н. Акимов ${ }^{+}$, А.Э. Климов ${ }^{+}$, С.В. Морозов ${ }^{*}$, С.П. Супрун ${ }^{+}$, В.С. Эпов ${ }^{+}$,

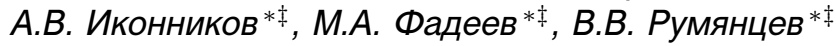 \\ + Институт фризики полупроводников им. А.В. Ржанова, \\ Сибирское отделение Российской академии наук, \\ 630090 Новосибирск, Россия \\ * Институт ффизики микроструктур Российской академии наук, \\ 630950 Нижний Новгород, Россия \\ ¥ Нижегородской государственный университет им. Н.И. Лобачевского, \\ 630950 Нижний Новгород, Россия \\ E-mail: klimov@isp.nsc.ru
}

(Получена 27 апреля 2016 г. Принята к печати 10 мая 2016 г.)

Представлены экспериментальные результаты исследований динамики фотопроводимости пленок PbSnTe:In c высоким содержанием SnTe и с соответствующим краем фундаментального поглощения вблизи 30 мкм при гелиевых температурах. Рассмотрены возможные причины гигантской (выше 2 порядков) отрицательной фотопроводимости образцов.

\section{1. Введение}

Ширина запрещенной зоны $\left(E_{g}\right)$ твердого раствора бинарных соединений $\mathrm{PbTe}$ и $\mathrm{SnTe}-\mathrm{Pb}_{1-x} \mathrm{Sn}_{x} \mathrm{Te}$ $(\mathrm{PbSnTe})$ - зависит от температуры $T$, параметра состава $x$ и при некотором его значении $x_{0}$ обращается в нуль [1] с инверсией зоны проводимости и валентной зоны в точке $L_{6}$ зоны Бриллюэна. Величина $x_{0}$ зависит от температуры и при $T=4.2 \mathrm{~K} x_{0} \approx 0.35$. Равновесная концентрация свободных носителей заряда электронов $n$ (дырок $p$ ) для любых составов нелегированного $\mathrm{PbSnTe}$, полученного с использованием различных технологий, остается высокой даже при гелиевых температурах. Это обусловлено электрически активными собственными точечными дефектами (вакансиями металлов или теллура) с малой энергией ионизации, концентрация которых высока в силу термодинамических особенностей кристаллизации $\mathrm{PbSnTe}$. Большие значения $n(p)$, помимо прочего, ведут к малым временам жизни неравновесных носителей заряда $\tau$. Так как $n(p)$ и $\tau$ определяют минимально достижимые темновые токи $p-n$-переходов, то фотоприемники фотодиодного типа с высокими параметрами удалось создать лишь в ограниченной области составов PbSnTe, а именно при $x \leq 0.18-0.20$. Красная граница чувствительности для этих составов соответствует длине волны $\lambda_{\max } \lesssim 12$ мкм. Одновременно большие величины $n_{0}\left(p_{0}\right)$ и малые $\tau$ в нелегированном $\mathrm{PbSnTe}$ не позволяют создавать на его основе эффективные фотосопротивления с параметрами, лучшими, чем параметры фотодиода.

Ситуация радикально меняется при введении в $\mathrm{PbSnTe}$ индия до концентраций $1-3 \mathrm{aT} \%$. Было обнаружено $[2,3]$, что в определенном диапазоне составов, который по различным литературным данным соответствует $x \approx 0.22-0.29$ [4], добавление индия ведет к резкому снижению равновесной концентрации носителей заряда с расположением уровня Ферми в запрещенной зоне. Одновременно происходит радикальное увеличение $\tau$, которое при $T \leq 20-30 \mathrm{~K}$ составляет миллисекунды и более, а при $T=4.2 \mathrm{~K}$ может превышать часы и сутки. В указанном интервале при увеличении $x$ уровень Ферми перемещается от дна зоны проводимости к потолку валентной зоны. Следовательно, при определенном составе он может быть расположен и вблизи середины запрещенной зоны, обеспечивая проводимость без освещения, близкую к собственной. Диапазон составов с $x \approx 0.22-0.29$ при $T=4.2 \mathrm{~K}$ соответствует ширине запрещенной зоны $E_{g} \approx 0.07-0.04$ эВ [1] и краю фундаментального поглощения $\lambda_{\max } \approx 18-31$ мкм. Эти свойства $\mathrm{PbSnTe}: \mathrm{In}$ открыли перспективы для создания на его основе эффективных глубокоохлаждаемых фотоприемников на основе фотопроводимости (ФП) и многоэлементных фотоприемных устройств (МФПУ) дальнего инфракрасного диапазона, которые были реализованы в [5].

Вместе с тем по-прежнему не вполне понятен механизм „стабилизации“ уровня Ферми внутри запрещенной зоны при введении в $\mathrm{PbSnTe}$ индия и то, насколько жестко это явление привязано к указанному диапазону составов с $\lambda_{\max } \approx 18-31$ мкм для тех или иных технологий получения слоев PbSnTe:In. Особый интерес вызывает исследование возможности получения пригодных для создания эффективных МФПУ тонких слоев $\mathrm{PbSnTe}: \mathrm{In}$ таких составов, для которых по крайней мере $\lambda_{\max } \geq 30-40$ мкм. Это связано с тем, что по целому ряду причин МФПУ этого диапазона на основе оптических межзонных переходов имеют существенные преимущества по сравнению с МФПУ, в которых используются примесные оптические переходы или болометрический эффект.

Целью данной работы было исследование особенностей фотоэлектрических явлений в пленках $\mathrm{PbSnTe}: \mathrm{In}$ 
с повышенным содержанием теллурида олова, соответствующим краю фундаментального поглощения $\sim 30$ мкм или более.

\section{2. Образцы и методика эксперимента}

Исследованные пленки PbSnTe:In были выращены методом молекулярно-лучевой эпитаксии (МЛЭ) на подложках как $\mathrm{BaF}_{2}$, так и $\mathrm{Si}$. Последние по ряду причин представляют особый интерес для технологий МФПУ большого формата. Типичная толщина пленок составляла 1-2 мкм. В обоих случаях ориентация подложек была (111). Более детально технология получения пленок описана, например, в $[5,6]$. Отметим, что на кремниевых подложках пленки PbSnTe: In выращивались с использованием буферных слоев $\mathrm{CaF}_{2} / \mathrm{BaF}_{2}$ толщиной несколько десятых долей микрометра [7]. Измерения фотоэлектрических свойств пленок проводились как на стандартных холловских структурах с размером рабочей области $0.02 \times 0.05$ мм, так и на структурах, представляющих собой два паянных индиевых контакта к пленке $\mathrm{PbSnTe}: \mathrm{In}$ с характерным расстоянием между ними $\sim 0.1 \mathrm{~cm}$.

Концентрация и подвижность носителей заряда определялись из измерений эффекта Холла в постоянном магнитном поле с индукцией $\sim 0.2$ Тл. Образцы располагались в парах гелия в экранированной от внешнего фонового излучения металлической камере. Динамика изменения фототока измерялась в этой же камере с использованием в качестве источника излучения миниатюрной лампы накаливания с вакуумированной стеклянной колбой. На лампу подавалось напряжение питания не более $1.3 \mathrm{~B}$ при номинальном напряжении питания лампы 12 В. Лампа располагалась вдали от образца на расстоянии $\sim 2.5 \mathrm{~cm}$.

Измерения спектральных зависимостей ФП были выполнены с использованием фурье-спектрометра Bruker Vertex 80v. В данном случае образец располагался на конце волноводной вставки, погружаемой в транспортный сосуд Дьюара с жидким гелием. В качестве источника излучения использовалось абсолютно черное тело (глобар) с температурой $1000^{\circ} \mathrm{C}$. Поглощение в атмосферном воздухе было исключено путем откачки внутренней полости спектрометра, отделенной от части волноводной вставки, погруженной в сосуд Дьюара, с помощью герметичного фильтра из прозрачного полиэтилена. Данный фильтр в сочетании с разными светоделителями обеспечивал возможность записи спектров в диапазоне длин волн 1.5-28 мкм (для светоделителя на основе $\mathrm{KBr}$ ) или 14-300 мкм (для светоделителя на основе майлара). Для приложения постоянного напряжения к образцу и усиления электрического сигнала использовался малошумящий токовый усилитель Stanford Research Systems SR-570 с возможностью компенсации постоянной составляющей тока.

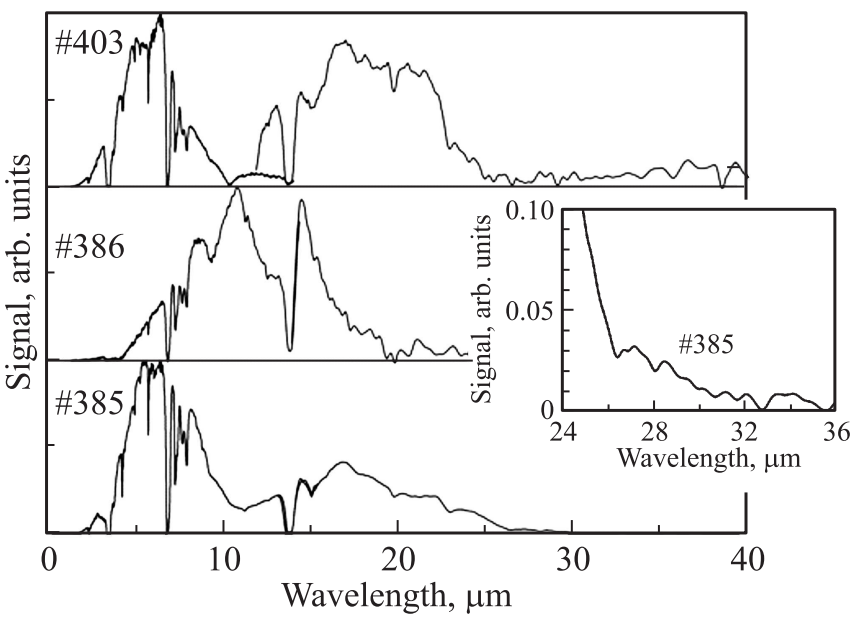

Рис. 1. Спектральные зависимости фотопроводимости образцов \# 385, 386 и 403 при $T=4.2 \mathrm{~K}$. На вставке - в увеличенном масштабе длинноволновой край спектра образца \# 385 .

\section{3. Экспериментальные результаты}

\section{1. Спектральные зависимости фотопроводимости}

На рис. 1 показаны спектральные зависимости ФП при $T=4.2 \mathrm{~K}$ для трех образцов: \#385, 386 и 403. Некоторые из их параметров приведены в таблице. Как видно из таблицы, все образцы имеют высокую подвижность электронов $(\mu)$ уже при азотной температуре, a ее максимальные значения в области $T=20-30 \mathrm{~K}$ достигают величин $30000-97000 \mathrm{~cm}^{2} \cdot \mathrm{B}^{-1} \cdot \mathrm{c}^{-1}$. Такие высокие значения подвижности наряду с данными по дифракции электронов на отражение, использовавшейся в процессе МЛЭ, подтверждают высокое кристаллическое качество получаемых по разработанной технологии слоев. Данные по составу пленок получены методом рентгеновского микроанализа. Для образца \#385 содержание индия не превышало ошибку измерений, т.е. было не более 0.1 ат\%. Проводимость и концентрация носителей заряда в образце \#386 слабо менялись ниже температуры жидкого азота. Состав этого образца не измерялся, поскольку пленки с такими свойствами не представляют интереса для создания фотоприемников.

Фоточувствительность пленок при $T=4.2 \mathrm{~K}$ контролировалась с использованием лампы накаливания (см. разд. 2). Она была высокой для образцов \#385 и 403 и сравнительно небольшой для \#386, в котором равновесная концентрация электронов велика.

Узкие линии в спектрах рис. 1 соответствуют положению линий поглощения оптических элементов фурьеспектрометра и измерительного оптического криостата (светоделители и оптические окна) и не являются особенностями собственно образцов. Как видно из рис. 1 , фоточувствительность превышает шум примерно до длин волн 24, 19 и 30 мкм для образцов \# 403, 386 и 385 


\begin{tabular}{c|c|c|c|c|c|c}
\hline $\begin{array}{c}\text { Номер } \\
\text { образца }\end{array}$ & $\begin{array}{c}\text { Подложка/ } \\
\text { толщина, мкм }\end{array}$ & $x$ & $\begin{array}{c}\text { Содержание } \\
\mathrm{In}, \text { ат\% } \%\end{array}$ & $\begin{array}{c}n, \mathrm{~cm}^{-3} / \mu, \mathrm{cm}^{2} \cdot \mathrm{B}^{-1} \cdot \mathrm{c}^{-1} \\
(T=77 \mathrm{~K})\end{array}$ & $\begin{array}{c}n, \mathrm{~cm}^{-3} \\
(T=20 \mathrm{~K})\end{array}$ & $\begin{array}{c}\text { Максимальная } \\
\text { подвижность } \mu(T), \\
\mathrm{cm}^{2} \cdot \mathrm{B}^{-1} \cdot \mathrm{c}^{-1}\end{array}$ \\
\hline$\# 385$ & $\mathrm{Si} / 2$ & 0.3 & - & $1.3 \cdot 10^{15} / 13200$ & $8.7 \cdot 10^{12}$ & $29400(T=35 \mathrm{~K})$ \\
$\# 386$ & $\mathrm{BaF}_{2} / 1.9$ & - & - & $1.6 \cdot 10^{16} / 30000$ & $1.6 \cdot 10^{16}$ & $97000(T=20 \mathrm{~K})$ \\
$\# 403$ & $\mathrm{BaF}_{2} / 1.3$ & 0.29 & 0.1 & $1.6 \cdot 10^{15} / 12000$ & $1.8 \cdot 10^{11}$ & $43000(T=20 \mathrm{~K})$
\end{tabular}

соответственно. Наибольший интерес представляют, как сказано во Введении, образцы с максимальной красной границей чувствительности, в данном случае образец \# 385; именно ему было уделено максимальное внимание в исследованиях, результаты которых приведены далее.

\section{2. Температурные зависимости концентрации и подвижности}

Температурные зависимости концентрации и подвижности электронов, $n(T)$ и $\mu(T)$, были измерены для всех трех образцов. Некоторые из значений приведены в таблице. Зависимости $n(T)$ и $\mu(T)$ образца \#385, данные по динамике ФП которого будут приведены далее, обладали следующими особенностями. Эффект Холла был измерен при $T \geq 20 \mathrm{~K}$. Ниже этой температуры сопротивление образца было слишком велико для корректных измерений. Концентрация электронов уменьшалась примерно от $n \approx 7 \cdot 10^{17} \mathrm{~cm}^{-3}$ при $300 \mathrm{~K}$ до $n \approx 1.3 \cdot 10^{15} \mathrm{~cm}^{-3}$ при $80 \mathrm{~K}$ и $n \approx 8.7 \cdot 10^{12} \mathrm{~cm}^{-3}$ при $20 \mathrm{~K}$. В области $T \approx 20-50 \mathrm{~K}$ поведение $n(T)$ описывается зависимостью, близкой к зависимости Аррениуса с энергией активации $\Delta E \approx 0.0094$ эВ. Выше $T=50 \mathrm{~K}$ зависимость $n(T)$ становится сильнее. Подвижность электронов растет с понижением температуры от комнатной до $T \approx 35 \mathrm{~K}$, где она максимальна (см. таблицу), а затем уменьшается, принимая значение $\mu \approx 10000 \mathrm{~cm}^{2} \cdot \mathrm{B}^{-1} \cdot \mathrm{c}^{-1}$ при $T=20 \mathrm{~K}$. Наличие максимума подвижности в области $T \approx 10-40 \mathrm{~K}$ достаточно типично для $\mathrm{PbSnTe}$. Особенности температурной зависимости подвижности при низких температурах объясняются рассеянием на ионизованных примесях [8]. В области $T \approx 50-300 \mathrm{~K}$ поведение $\mu(T)$ близко к степенно́й зависимости вида $\mu \propto T^{-5 / 2}$, также типичной для $\mathrm{PbSnTe}[8]$. Необычной особенностью этого образца является отрицательное магнетосопротивление в области $T \leq 30 \mathrm{~K}$ с относительным уменьшением сопротивления примерно на $10-15 \%$ при $B \approx 0.2$ Тл вблизи температуры жидкого гелия. При $T \geq 30 \mathrm{~K}$ наблюдалось обычное положительное магнетосопротивление.

\section{3. Динамика фотопроводимости}

Динамика изменения тока при включении и выключении освещения была измерена для образца \# 385. Структура для измерений представляла собой два паянных индиевых контакта к пленке PbSnTe:In, разделенных

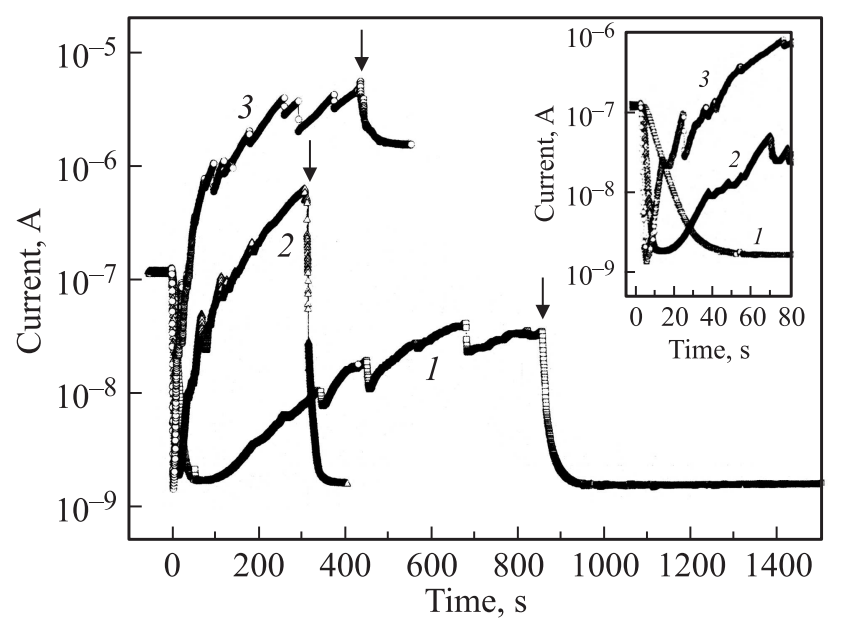

Рис. 2. Временнб́е зависимости тока в образце \#385 при $T=4.2 \mathrm{~K}$. Напряжение смещения $U=4.6(1)$ и $3.8 \mathrm{~B}(2,3)$. Напряжение и ток источника освещения, В/мА: 0.345/1.0 (1), $0.65 / 1.32(2), 1.26 / 4.6(3)$. На вставке - начальный участок зависимостей. Освещение включено при $t=0$, стрелками показаны моменты выключения освещения.

зазором $\sim 0.2 \mathrm{~cm}$. Отсутствие заметных токов утечки в кремниевую подложку проверялось с использованием третьего контакта к пленке PbSnTe:In, отделенного от измеряемого участка поверхностью кремния, на которой пленка PbSnTe:In отсутствовала. Методика измерения фототока описана в разд. 2. На рис. 2 приведены зависимости изменения тока $I$ через образец при $T=4.2 \mathrm{~K}$, при напряжении смещения $U=4.6 \mathrm{~B}$ для кривой 1 и 3.8 В для кривых 2, 3 при трех режимах освещения (см. подпись к рисунку). Перед каждым измерением температура образца поднималась выше температуры жидкого азота, после чего образец вновь охлаждался и измерялись временны́е зависимости тока. Момент включения освещения для всех кривых соответствует моменту времени $t=0$, моменты выключения освещения показаны стрелками. Из рисунка видно, что после включения освещения наблюдается сравнительно быстрое уменьшение тока примерно на 2 порядка - отрицательная фотопроводимость (ОФП) - с последующим его увеличением.

На рис. 3 приведены начальные участки зависимостей $y(t)=\left[I(t)-I_{\min }\right] / I_{t=0}$ в полулогарифмическом масштабе, где $I_{\min }$ - минимальные значения тока, достигаемые после включения освещения, $I_{t=0}$ - значения тока 


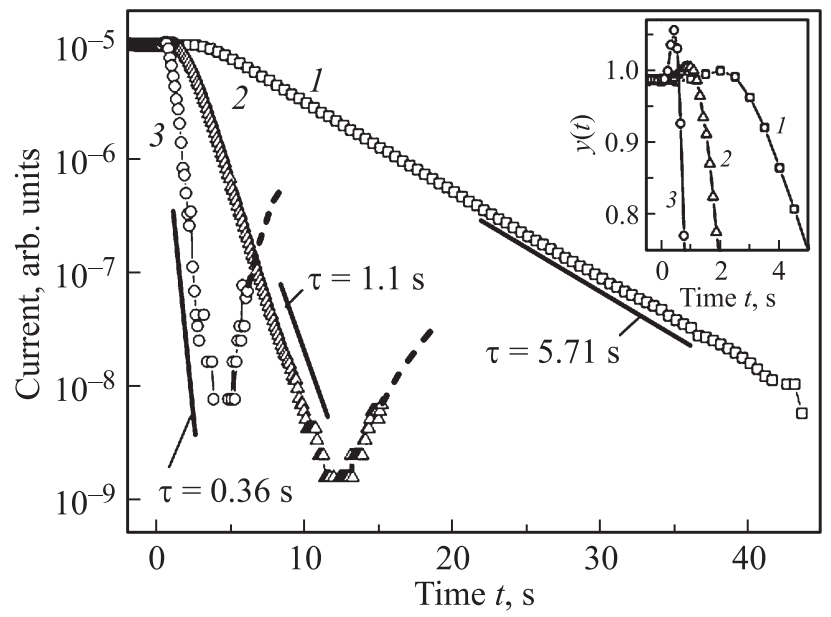

Рис. 3. Начальные участки зависимостей вида $y(t)=$ $=\left[I(t)-I_{\min }\right] / I_{t=0}$, где $I_{\min }-$ минимальные значения тока (см. рис. 2), $I_{t=0}-$ ток при $t=0$. Обозначение кривых аналогичны обозначениям на рис. 2. Отрезки прямых соответствуют зависимостям вида $\propto e^{-t / \tau}$, значения $\tau$ указаны. Штриховыми кривыми $(2,3)$ показаны направления дальнейшего роста тока со временем (см. рис. 2). На вставке - начальные участки $y(t)$ в линейном масштабе.

перед включением освещения. Как видно из рисунка, уменьшение тока сразу после включения освещения от его начальной величины к некоторому минимальному значению происходит по экспоненциальному закону в диапазоне изменения $y(t)$ до 3-4 порядков величины. Постоянная времени $\tau$ для этих зависимостей уменьшается при увеличении интенсивности освещения (напряжения на лампе). Сразу после включения освещения наблюдаются кратковременные небольшие „всплески“ тока, относительные величины которых составили примерно $0.016,0.020$ и 0.062 от тока до освещения для зависимостей $1,2,3$ соответственно.

На рис. 4 показана динамика изменения тока в виде той же функции $y(t)$ после выключения освещения. Выключение освещения соответствует моментам, указанным стрелками на рис. 2, но временны́е оси для зависимостей на рис. 4 сдвинуты так, что выключение освещения для всех трех кривых на рисунке соответствует моменту времени $t=0$. Видно, что, в отличие от участков ОФП после включения освещения, для каждой из зависимостей динамика уменьшения тока не описывается единой экспонентой, хотя и наблюдаются отдельные участки с разными наклонами, показанными на рисунке отрезками прямых. Кроме этого, на зависимостях имеются дополнительные особенности. Они наиболее заметны в интервале времени 0-20с и проявляются в виде „изломов“ (кривая 1), „изломов“, ступеньки и „провала“ (кривая 2), „провала“ и „выбросов тока“ (кривая 3).

Таким образом, ОФП наблюдается на начальных участках временны́х зависимостей в виде резкого (примерно на 2 порядка) уменьшения тока (рис. 2). Были из- мерены вольт-амперные характеристики (BAX) образца до освещения („низкоомный“ режим) и после выключения освещения („высокоомный“ режим). Последний сохранялся в течение длительного времени, многократно превышавшего время измерения ВАХ. На рис. 5 приведены $\mathrm{BAX} I(U)$ для обоих режимов. Видно, что в „низкоомном“ режиме до освещения ВАХ имеет слабую нелинейность и на использованных скоростях развертки напряжения гистерезис BAX практически отсутствует. В „высокоомном“ режиме ВАХ имеет выраженный нелинейный характер и сильный гистерезис. При этом в

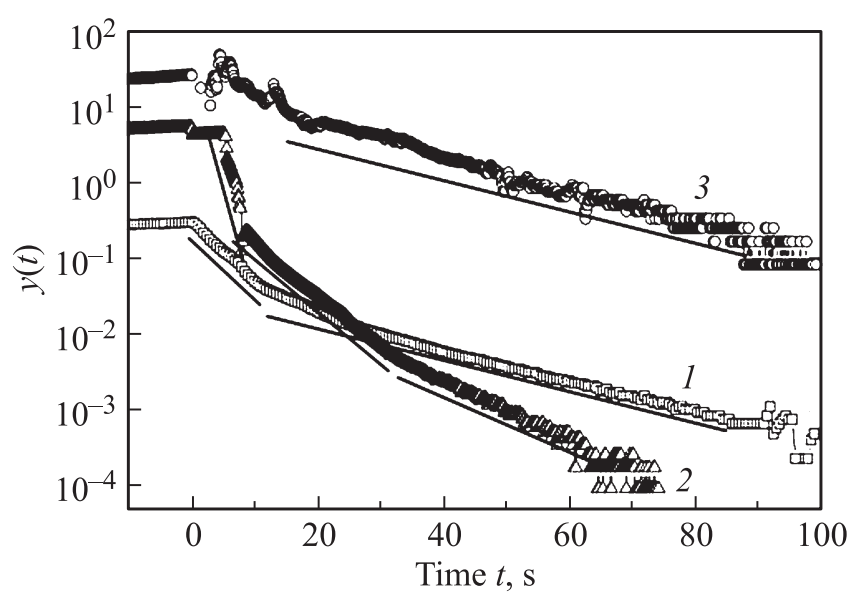

Рис. 4. Временнб́е зависимости величины $y(t)=$ $=\left[I(t)-I_{\min }\right] / I_{t=0} . \quad I_{t=0}-$ ток при $t=0$ на рис. 2 и 3. Обозначения кривых аналогичны обозначениям на рис. 2,3 . Здесь освещение выключено при $t=0$, что соответствует временам, показанным стрелками на рис. 2. $I_{\min }-$ минимальные значения тока, достигаемые после выключения освещения.

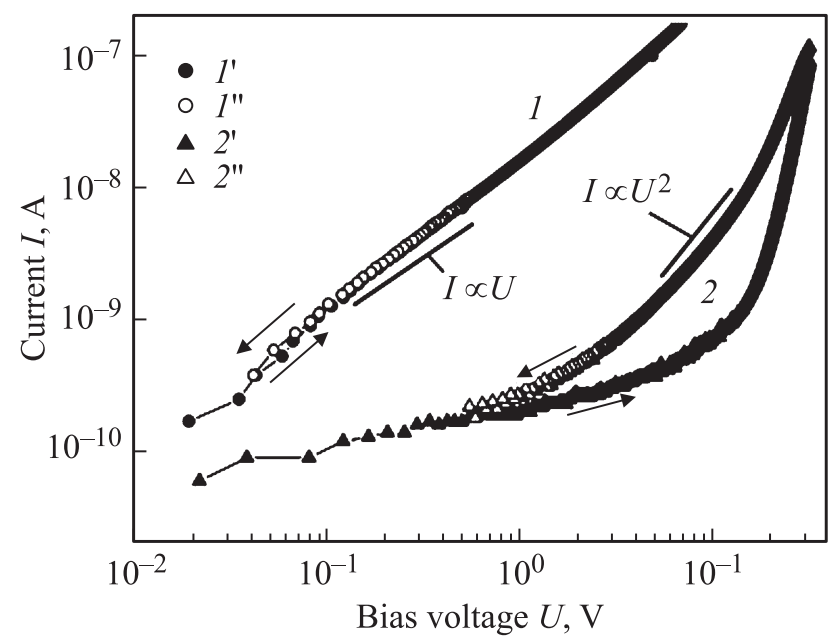

Рис. 5. ВАX $I(U)$ образца \# 385 при $T=4.2 \mathrm{~K}$ до освещения в „низкоомном“ состоянии (1) и после освещения в „высокоомном“ состоянии (2). $1^{\prime}, 2^{\prime}$ (стрелки вправо) соответствуют увеличению напряжения, $1^{\prime \prime}, 2^{\prime \prime}$ (стрелки влево) - уменьшению. Отрезки прямых соответствуют линейной и квадратичной зависимостям тока от напряжения. Скорость изменения $U$ равнялась 0.025 (1) и $0.083 \mathrm{~B} / \mathrm{c}(2)$. 
областях напряжений $U \leq 10-15 \mathrm{~B}$ (при увеличении $U$ ) и $U \leq 2-4$ В (при уменьшении $U$ ) зависимость тока от напряжения сублинейна, а в области больших напряжений суперлинейна.

Кроме этого, были проведены измерения динамики фототока при повышенной температуре, $T=12.9 \mathrm{~K}$. Основные результаты этих измерений заключаются в следующем. При этой температуре также после включения освещения наблюдается ОФП с уменьшением тока при тех же интенсивностях излучения в $30-60$ раз. Однако, в отличие от измерений при $4.2 \mathrm{~K}$, при $T=12.9 \mathrm{~K}$ уменьшение тока со временем описывается не одной, а двумя экспоненциальными зависимостями. Их постоянные времени оказались больше, чем при $T=4.2 \mathrm{~K}$, и составили $\sim 10, \sim 50$ с (режим освещения соответствует кривой 1 на рис. $3, \tau \approx 5.71 \mathrm{c})$ и $\sim 1.7, \sim 26 \mathrm{c}$ (режим освещения соответствует зависимости 3 на рис. $3, \tau \approx 0.36 \mathrm{c}$ ). При этом увеличения тока в начальный момент времени, как на вставке к рис. 3, не наблюдалось.

\section{4. Обсуждение результатов}

Уменьшение проводимости полупроводника в результате освещения известно давно и называется отрицательной фотопроводимостью $[9,10]$. В частности, к ОФП относят и инфракрасное гашение - уменьшение ФП, вызванной коротковолновым освещением, под воздействием более длинноволнового излучения. Однако в этом случае ФП уменьшается до значения, не меньшего проводимости без освещения. В общем случае, как и в настоящей работе, при ОФП проводимость может становиться меньше равновесной проводимости полупроводника. Известны два основных подхода для объяснения ОФП, заключающиеся в рассмотрении либо однородного образца, либо образца, включающего в себя те или иные неоднородности. К первому относится модель, развитая Штокманом [11]. В рамках этой модели ОФП наблюдается при энергии квантов, меньшей ширины запрещенной зоны. Схематично на примере полупроводника электронного типа проводимости суть модели можно сформулировать следующим образом: освещение ведет к переходу электронов из валентной зоны на малозаселенные в исходном состоянии уровни, расположенные выше уровня Ферми. Образующиеся при этом дырки рекомбинируют с электронами, находящимися на другом уровне, расположенном недалеко от потолка валентной зоны и заполненном в исходном состоянии. Модель предполагает, что для этого уровня сечения захвата электронов и дырок близки по величине. Таким образом, рекомбинация идет одновременно с захватом на уровень электронов из зоны проводимости. Как результат, концентрация электронов в зоне проводимости снижается на величину, равную концентрации электронов, захваченных на верхний уровень, время жизни на котором может быть достаточно велико. По сути в двухуровневой модели Штокмана происходит „перекачивание“ электронов из зоны проводимости на уровень вблизи ее дна, имеющий малое сечение захвата, через уровень вблизи потолка валентной зоны с использованием оптических переходов за краем фундаментальной области поглощения. Последнее является принципиальной особенностью данной модели.

Типичным примером неоднородных полупроводников, в которых может проявляться ОФП, являются слоистые структуры, содержащие либо отдельные гетеропереходы, либо их набор - например, многослойные квантовые ямы и сверхрешетки. Так, авторы [12] при определенных условиях наблюдали ОФП со сравнительно небольшой амплитудой в многослойных квантовых ямах $\mathrm{Pb}_{1-x} \mathrm{Sn}_{x} \mathrm{Te} / \mathrm{PbTe} / \mathrm{BaF}_{2}$, а авторы [13] - гигантскую ОФП в структурах $p$-типа проводимости с квантовыми ямами $\mathrm{GaAs}$ в матрице $\mathrm{AlGaAs}$ с модуляцией уровня легирования. Для объяснения ОФП в неоднородных структурах используются представления о генерации электронно-дырочных пар в более широкозонном полупроводнике с последующим их пространственным разделением во встроенных электрических полях. Наблюдаемое в [13] уменьшение проводимости почти в 100 раз по отношению к равновесной объясняется тем, что генерируемые при оптических межзонных переходах в широкозонном AlGaAs электроны, двигаясь в узкозонный слой GaAs, рекомбинируют в нем с равновесными дырками, уменьшая проводимость этого слоя. В свою очередь генерируемые излучением в широкозонном слое AlGaAs неравновесные дырки остаются захваченными на уровни в нем и не участвуют в проводимости. Подчеркивается, что такой процесс может вести к сильному уменьшению общей проводимости структур, если время захвата дырок в AlGaAs велико по сравнению с временем рекомбинации электронов в GaAs.

Измерения при $T=300 \mathrm{~K}$ спектра пропускания колбы лампы, использованной в качестве источника излучения в наших экспериментах, показали, что ее максимальная длина волны излучения не превышает 5 мкм (полуспад пропускания находится при $\lambda \approx 4.5$ мкм). Таким образом, в эксперименте энергия квантов была существенно больше ширины запрещенной зоны образцов (см. рис. 1), и модель Штокмана в нашем случае не подходит. В отличие от [12] и [13], в исследованных нами образцах пространственные неоднородности не создавались искусственно. Вместе с тем в [14] отмечалось, что при кристаллизации твердых растворов $\mathrm{PbSnTe}$ вообще и $\mathrm{PbSnTe}: \mathrm{In}$ в частности при определенных условиях возможно появление включений с другим фазовым составом, которые и могут играть роль вышеупомянутых пространственных неоднородностей.

Допустим, что в нашем случае в узкозонной матрице имеются более широкозонные пространственно изолированные включения. Тогда зонную диаграмму образца можно схематически представить так, как это показано на рис. 6.

До освещения (слева на схеме) проводимость определяется более узкозонной матрицей І. При включении 


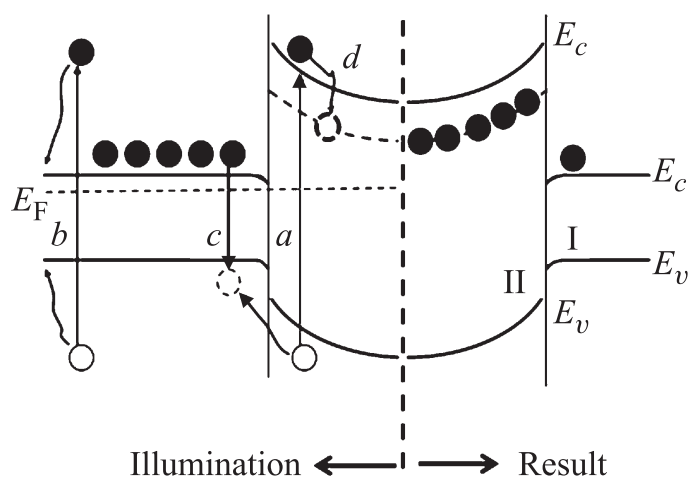

Рис. 6. Зонная диаграмма для объяснения ОФП в $\mathrm{PbSnTe}: \mathrm{In}$ с включениями широкозонной фазы. Слева от вертикальной штриховой линии - ситуация до и при освещении, справа после освещения.

освещения с энергией квантов, превышающей ширину запрещенной зоны и в области I, и в области II, генерация электронно-дырочных пар идет в обеих областях (переходы $a$ и $b$ ). Однако при этом обычная ФП в области I может оставаться незначительной, если темп межзонной рекомбинации достаточно велик (время рекомбинации мало), и проявляться лишь в начале освещения. Генерируемые излучением электронно-дырочные пары в области II разделяются встроенным электрическим полем. При этом дырки переходят в область I и рекомбинируют с электронами, уменьшая их концентрацию (переход $c$ ). Электроны в области II захватываются на уровни в запрещенной зоне с большими временами жизни. В результате реализуется ситуация, показанная справа (изменение изгиба зон, связанное с пространственным разделением заряда, на схеме не отображено). Таким образом, аналогично модели Штокмана происходит „перекачивание“ электронов из зоны проводимости на уровни захвата с уменьшением проводимости образца в целом. При этом уменьшение числа участвующих в проводимости электронов в области I равно числу захваченных на уровни электронов в области II. Разница с моделью Штокмана заключается в том, что в этом случае энергия квантов больше ширины запрещенной зоны, а проводящая область I пространственно отделена от области с ловушками II.

Рассмотрим теперь качественное соответствие модели результатам, наблюдаемым в эксперименте. На вставке к рис. 3 на начальном участке виден всплеск тока (обычная ФП), который в модели является следствием незначительного увеличения проводимости области I непосредственно после включения освещения. Далее на рис. 3 наблюдаются ярко выраженные участки ОФП с экспоненциальной зависимостью от времени, причем постоянная времени уменьшается с увеличением интенсивности освещения. В рамках рассматриваемой модели это объясняется следующим образом. В простейшем случае только одного типа уровня захвата в области II уравнение для концентрации электронов на нем $m$ можно записать в виде

$$
\frac{d m}{d t}=\alpha n_{\mathrm{II}}\left(N_{t}-m\right)-\beta m
$$

где $n_{\mathrm{II}}$ - концентрация электронов в области II, $N_{t}-$ концентрация соответствующих центров захвата там же. Решение этого уравнения имеет экспоненциальный вид с постоянной времени $\tau=1 / \alpha n_{\mathrm{II}}$,

$$
m(t)=N_{t}\left(1-e^{t / \tau}\right)
$$

в том случае, когда можно пренебречь вторым слагаемым в уравнении (1), отвечающим за обратные переходы электронов с уровня в зону проводимости, а величина $n_{\text {II }}$ слабо меняется во времени. Первое условие выполняется для глубоких уровней захвата с энергией ионизации $\Delta E_{t} \gg k T$. Выполнение второго условия в рамках рассматриваемой модели возможно по следующим соображениям. В однородном полупроводнике захват электронов на ловушки в условиях сохранения электронейтральности объема сопровождается ростом концентрации неравновесных дырок и, как следствие, уменьшением со временем величины $n_{\text {II при постоянном }}$ темпе оптической генерации $g$. В нашем случае электронейтральность нарушается и генерируемые светом в области II дырки уходят в область I. Вследствие этого

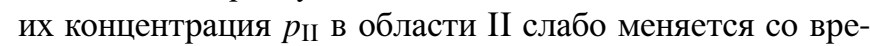
менем, откуда и следует слабое изменение $n_{\mathrm{II}}$. При этом постоянная времени $\tau$ в (2) оказывается обратно пропорциональной концентрации $n_{\mathrm{II}}$, которая увеличивается с увеличением $g$ (интенсивности освещения). В рамках рассматриваемой модели с такой же скоростью, как и величина $m(t)(2)$, будет уменьшаться концентрация электронов $n_{\mathrm{I}}(t)$ в области I, т.е. ее зависимость от времени будет задаваться соотношением

$$
n_{\mathrm{I}}(t)=n_{0 \mathrm{I}}-N_{t}\left(1-e^{1 / \tau}\right),
$$

описывающим экспоненциальное уменьшение концентрации носителей заряда и проводимости образца в целом до некоторой постоянной величины, как это и наблюдается в эксперименте для тока ОФП.

Наконец, обратимся к некоторым экспериментальным данным, связанным с измерениями при повышенной температуре. Заметное увеличение постоянной времени на участках ОФП в $2-5$ раз и более при $T=12.9 \mathrm{~K}$ по сравнению с $T=4.2 \mathrm{~K}$, выглядящее несколько необычно, в рассматриваемой модели объясняется тем, что с ростом температуры уменьшается $n_{\mathrm{II}}$ как результат уменьшения времени жизни относительно межзонной рекомбинации при различных ее механизмах.

Необходимо отметить, что рассмотренная модель, привлекающая представления об уровнях захвата в широкозонных включениях в узкозонной матрице, допускает и наличие соответствующих уровней захвата в узкозонной области, проявляющих себя в различных релаксационных процессах, описанных в многочисленных 
работах и обзорах, - см., например, [4]. Следствием присутствия таких уровней, распределенных по энергии, могут быть и специфические особенности релаксации обычной ФП после выключения освещения (рис. 4), и особенности ВАХ (рис. 5), которая в условиях низкой проводимости определяется токами инжекции из контактов, ограниченными пространственными зарядами. Для таких ВАX характерен гистерезис, связанный с медленной перезарядкой уровней, и различные особенности, определяемые сложным энергетическим спектром уровней.

\section{5. Заключение}

В заключение отметим следующее. На первый взгляд, наличие неоднородностей в образцах выглядит отрицательным фактором, который может ограничивать потенциальное использование таких структур при разработке фотоприемников. С другой стороны, как следует из приведенных результатов, эти же включения позволяют с высокой точностью, варьируя интенсивность и длительность предварительного освещения высокоэнергетичными квантами, минимизировать „условно темновую“" квазистационарную проводимость узкозонных областей структуры. Поскольку такое состояние с низкой проводимостью может существовать достаточно долго после выключения предварительного освещения, это открывает интересные перспективы для использования именно таких структур в качестве основы для сверхдлинноволновых фотоприемников на основе $\mathrm{PbSnTe}: \mathrm{In}$ c $x>0.3$ и $\lambda_{\max }>30-40$ мкм, для которых получение однородных по составу пленок с низкой равновесной темновой проводимостью вызывает большие сложности.

\section{Список литературы}

[1] W.W. Anderson. IEEE J. Quant. Electron., 13 (7), 532 (1979).

[2] Б.А. Волков, О.А. Панкратов. ДАН СССР, 255 (1), 93 (1980).

[3] И.А. Драбкин, Б.Я. Мойжес. ФТП, 15 (4), 625 (1981).

[4] Б.А. Волков, Л.И. Рябова, Д.Р. Хохлов. УФН, 172 (7), 875 (2002).

[5] А.Э. Климов, В.Н. Шумский. В кн.: Матричные фотоприемные устройства (Н., Наука, 2001).

[6] E.V. Fedosenko, A.E. Klimov, D.V. Krivopalov. Appl. Surf. Sci., 78 (4), 413 (1994).

[7] А.Н. Акимов, А.В. Беленчук, А.Э. Климов, М.М. Качанова, И.Г. Неизвестный, С.П. Супрун, О.М. Шаповалов, В.Н. Шерстякова, В.Н Шумский. Письма ЖТФ, 35 (11), 88 (2009).

[8] Ю.И. Равич, Б.А. Ефимова, И.А. Смирнов. Методы исследования полупроводников в применении к халькогенидам свинца PbTe, PbSe, PbS (М., Наука, 1968).

[9] Р. Бьюб. Фотопроводимость твердых тел (М., Мир, 1962).

[10] А. Роуз. Основы теории фотопроводимости (М., Мир, 1966).

[11] F. Stöckman. Zs. Phys., 143, 348 (1955).
[12] I.I. Zasavitsky, B.N. Matsonashvili, V.T. Trofimov. Semicond. Sci. Technol., 8, 345 (1993).

[13] R.A. Höpfel. Appl. Phys. Lett., 52 (10), 801 (1988).

[14] Г.А. Калюжная, К.В. Киселева. Тр. ФИАН им. П.Н. Лебедева (М., Наука), 177, 5 (1987).

Редактор Л.В Шаронова

\section{Giant negative photoconductivity of PbSnTe: In films with cutoff wavelength near $30 \mu \mathrm{m}$.}
A.N. Akimov ${ }^{+}$, A.E. Klimov + , S.V. Morozov* S.P. Suprun ${ }^{+}$, V.S. Epov ${ }^{+}$, A.V. Ikonnikov* ${ }^{* \ddagger}$, M.A. Fadeev*‡, V.V. Rumyantsev*¥

+ Institute for Semiconductor Physics, Siberian Branch of Russian Academy of Sciences, 630090 Novosibirsk, Russia

* Institute for Physics of Microstructures,

Russian Academy of Sciences,

603950 Nizhny Novgorod, Russia

$\ddagger$ Lobachevsky State University of Nizhny Novgorod, 603950 Nizhny Novgorod, Russia

Abstract We present experimental results on photoconductivity dynamics in SnTe-rich PbSnTe:In films with the long-wavelength edge of interband transitions near $30 \mu \mathrm{m}$ at liquid helium temperature. The possible reasons for giant (more than two orders of magnitude) negative photoconductivity of samples are discussed. 